\title{
Proximal wrist extensor tendinopathy
}

\author{
Joseph Ihm
}

Published online: 2 November 2007

(C) Humana Press 2007

\begin{abstract}
Proximal wrist extensor tendinopathy, which is also known as tennis elbow, is pain at or just distal to the lateral humeral epicondyle within the proximal wrist extensor tendon. It occurs commonly in certain athletes but can also occur in people with jobs that require repetitive movements of the hand and upper limb. In most cases the tendon involved shows no signs of inflammation or tendonitis, but instead shows fibroblasts, vascular hyperplasia, and disorganized collagen. Diagnosis is often made by history and physical exam alone. Most people respond to conservative measures including activity modification, analgesics, manipulation of tissue, and exercise. In some cases, an injection of corticosteroid or botulinum toxin may be used. Surgery is rarely needed.
\end{abstract}

Keywords Tennis elbow - Elbow pain ·

Lateral epicondylitis

\section{Introduction}

Proximal wrist extensor tendinopathy is a condition most commonly known as tennis elbow, which is pain at or just distal to the lateral humeral epicondyle within the proximal wrist extensor tendon. Many diagnoses have been used to describe this disorder including tendinosis of the lateral elbow, lateral epicondylitis, lateral epicondylalgia, or lateral epicondylopathy. Major first described this disorder in 1883 as a condition causing lateral elbow pain in tennis players [1]. While up to $40 \%$ of tennis players may experience this condition, it affects as many as $15 \%$ of workers

J. Ihm ( $\square)$

Rehabilitation Institute of Chicago, Chicago, IL, USA

e-mail: jihm1@ric.org in highly repetitive hand task industries, and can result in an average duration of 12 weeks of absenteeism in as many as $30 \%$ of all those afflicted [2]. In a Finnish population study, the prevalence was $1.3 \%$ of people aged $30-64$ years old and was more likely to occur in people who smoke, have type 2 diabetes, and are involved in occupations requiring repetitive movements of the arms and those handling loads heavier than $20 \mathrm{~kg}$ [3]. Proximal wrist extensor tendinopathy occurs much more frequently than medial-sided elbow pain, with reported ratios ranging from 4:1 to $7: 1$ [1]. This article will discuss the pathophysiology, diagnosis, and treatment of the disorder.

\section{Pathophysiology}

Typically, after soft tissue trauma, there are three phases of healing [4]: inflammation, repair, and remodeling. The individual phases do not occur exclusively, and there is significant overlap. The first phase is the inflammatory phase and can last up to several days. In this phase, inflammatory mediators signal immune cells to migrate to the area to clean up debris. Once at the site of injury, immune cells release cytokines that bring fibroblasts to the area to begin the repair phase. The repair phase begins during the inflammatory phase and can last weeks. In this phase, fibroblasts lay down collagen according to the tension placed across the area being healed. Over time remodeling occurs, and the tissue reorganizes and becomes stronger as a result of an expansion of the cross-bonds between collagen fibers and replacement of the collagen fibers themselves.

While the term lateral epicondylitis is used most often for wrist extensor tendon pain in the lateral elbow, an inflammatory process is present probably only in the very 
early stages of the disorder. The pathology is considered to originate within the extensor carpi radialis brevis musculotendinous unit [1], though up to one third of patients have involvement in the origin of the extensor digitorum communis [5]. Histopathological studies have shown that specimens of tendon obtained from areas of chronic overuse, such as with proximal wrist extensor tendinopathy, do not contain large number of macrophages, lymphocytes, or neutrophils [5]. Normal tendon contains bundles of type-I collagen that are arranged in line with the long axis of the tendon with a sparse number of fibroblasts. In contrast, in patients with proximal wrist extensor tendinopathy, there appears to be a degenerative process that is characterized by the presence of fibroblasts, vascular hyperplasia, and disorganized collagen, which is commonly called tendinosis [5]. These changes occur in other tendinopathies including Achilles, patellar, and supraspinatus tendinopathy.

\section{Diagnosis}

Patients often present with symptoms that begin insidiously and can become worse with time. Many will not be able to describe an inciting event, but may be able to remember the first time the symptoms were felt. Prior to presenting to a health care professional, patients often will attempt to rest the area and may use ice and/or NSAIDs to try to alleviate the pain. Unfortunately, the pain often returns once regular use of the hand or forearm is resumed. Patients generally point to the lateral elbow as the source of their pain, but sometimes will point along the majority of the wrist extensor muscle mass. When the pain becomes more severe, symptoms often are felt with gripping activities, shaking hands, turning doorknobs, or lifting objects with the forearm pronated. Pain at rest occurs only in the most severe cases. Three physical exam tests can assist in the diagnosis [6]. The first is Cozen's test, which involves asking the patient to make a fist, pronate the forearm, and extend the wrist toward radial deviation, while the examiner resists this motion (Fig. 1). Pain in the area of the lateral epicondyle indicates a positive test. Another test is Mill's test, in which the examiner palpates the lateral epicondyle with one hand, and with the other, pronates the patient's forearm, flexes the wrist fully, and extends the elbow (Fig. 2). Pain at the lateral epicondyle indicates a positive test. A third test involves having the examiner resisting extension of the long finger of the patient's symptomatic side (Fig. 3). Reproduction of the patient's pain in the area of the lateral epicondyle indicates a positive test.

Some have attempted to describe clinical criteria for diagnosing proximal wrist extensor tendinopathy, listing

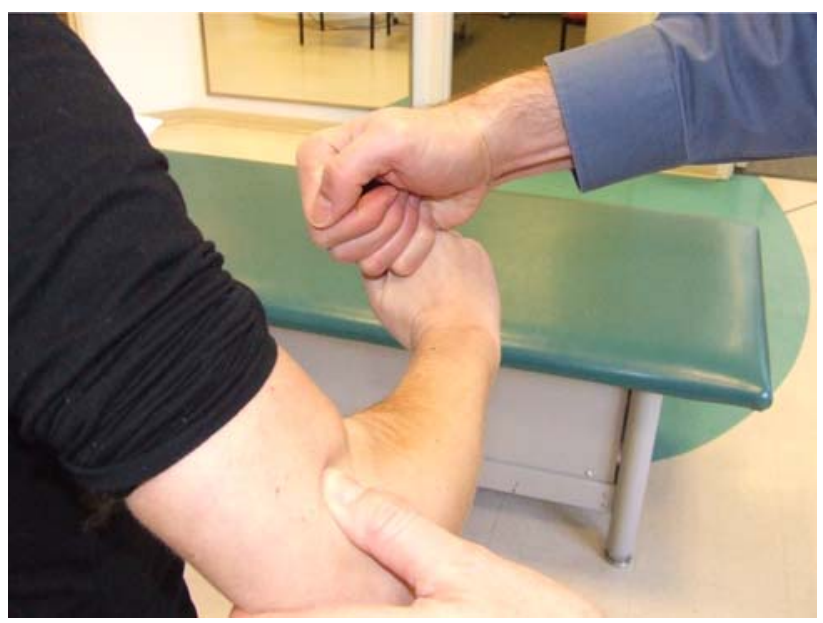

Fig. 1 Cozen's test

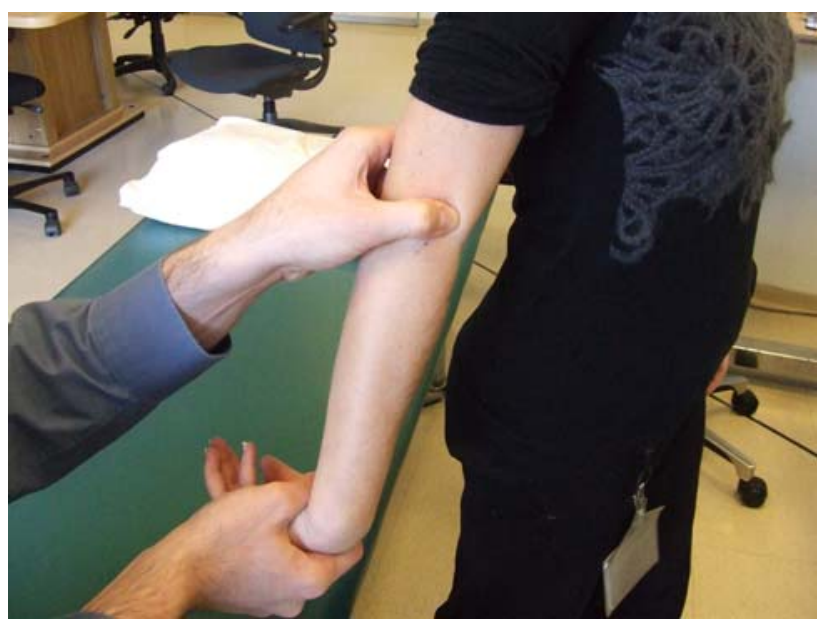

Fig. 2 Mill's test

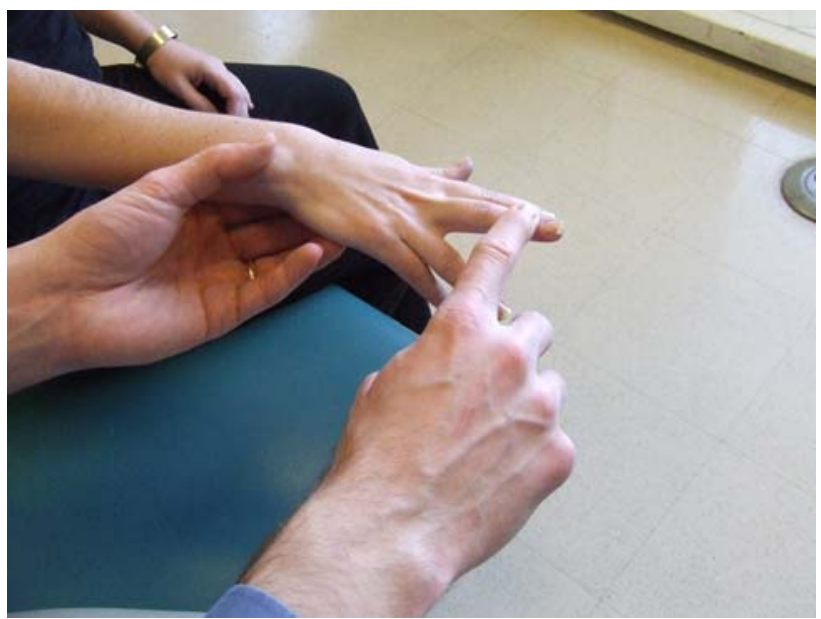

Fig. 3 Resisted finger extension 
three findings that should be present: lateral epicondylar pain, epicondylar tenderness, and pain on resisted extension of the wrist [7]. Some suggest that grip strength should be tested to see if pain is reproduced during gripping or to compare strength to the unaffected side [1]. With proximal wrist extensor tendinopathy, there is usually no pain at rest, and no numbness or tingling. If numbness or tingling is present, then one must consider a neurologic cause for the pain, such as a cervical radiculopathy or entrapment radial neuropathy in the elbow. Other diagnoses that may be considered, especially in athletes, include radiocapitellar disorders, osteochondritis dessicans, lateral collateral ligament incompetency, and plica band syndrome.

Routine radiographs of the elbow can assess for osteoarthritis of the elbow or calcific tendonitis of the wrist extensor mass, but often these are normal. Tendinous calcifications may be present in upto $7 \%$ of cases [1]. Advanced imaging is generally not needed to manage this condition. When a patient's symptoms are refractory to treatment, a diagnostic ultrasound or an MRI may be useful to assess the wrist extensor tendons to confirm the diagnosis of proximal wrist extensor tendinopathy and rule out other causes of pain.

\section{Treatment}

\section{Nonoperative}

Nonoperative treatment is generally effective and includes ice, nonsteroidal anti-inflammatory drugs (NSAIDs), bracing (e.g., with a forearm strap), activity modification, physical therapy, and injections. Patients often try rest, ice, modified activity, a forearm strap, or NSAIDs before seeking care, but these may provide only short-term relief. Given that the pathologic process is usually not inflammatory, the use of NSAIDs only provides relief of pain. Studies using NSAIDs to treat individuals who have a tendinopathy show minimal, if any improvement of pain [8]. Animal and in vitro studies have not generated consistent data to suggest that NSAIDs are appropriate or beneficial in tendinopathy [8]. While icing may be recommended, a recent pilot study showed that a 4-week physical therapy exercise program was effective in decreasing pain in lateral epicondylitis, regardless of whether or not ice was used [9].

While multiple meta-analyses have been done to evaluate the effectiveness of physical interventions on proximal wrist extensor tendinopathy, the most recent is by Bisset et al. [2]. In this meta-analysis, the diagnosis of tennis elbow or lateral epicondylitis was given to those with lateral elbow pain that increased on palpation and/or had reproduction of pain on resisted wrist extension. While 74 randomized controlled trials with physical interventions for lateral epicondylitis were identified, only 28 studies were of sufficient methodological quality to be included for analysis. Based on those studies that were included, it was found that manipulation techniques (including crossfriction massage), exercise, and acupuncture provided significant short-term relief. Studies that evaluated the efficacy of orthoses (including a forearm strap), taping, laser therapy, extracorporeal shock wave therapy, electromagnetic field and ionization, ultrasound, and phonophoresis either did not demonstrate a significant effect or showed an inconsistent effect on outcomes. However, given the small number of studies that met criteria for evaluation, more studies may be needed to determine the efficacy of physical interventions for this condition.

Only one study that involved exercise therapy in the meta-analysis met criteria to be evaluated, whereas all the other physical interventions had two or more studies that met criteria. A recent review on the effectiveness of eccentric exercise on chronic tendinopathy, including patellar and Achilles tendinopathy, found three studies on lateral epicondylitis that met criteria for evaluation [10]. Of the studies evaluated, there is conflicting evidence on the effect of eccentric training on outcomes. Outcomes measured including assessment of pain, function and return to activity. One investigator, Alfredson, has done multiple studies on tendinopathy has evaluated the efficacy of eccentric training on Achilles tendinopathy, and did so while encouraging reproduction of the tendon pain during the therapy sessions. A goal of reproducing patients' pain during rehabilitative exercise is a paradigm shift in the treatment of musculoskeletal disorders, since, often patients' pain symptoms are avoided or minimized during exercise sessions. He has reported successful outcomes using this method [11]. While Achilles tendinopathy appears to have a similar histologic picture as proximal wrist extensor tendinopathy [5], the function of the Achilles tendon is much different, so comparing treatments for these two conditions may be limited. Studies done to evaluate the effectiveness of eccentric strength training demonstrate that it can take weeks to be completely effective [11]. Further research will clarify the exact role of exercise therapy and determine the specific exercises that help those with proximal wrist extensor tendinopathy.

Injections should be considered in patients if above interventions have not provided relief, or in those whose pain on presentation is more inflammatory or debilitating. The most common injection performed is a corticosteroid injection, which is injected at the maximal site of tenderness. A recent review on the effectiveness of corticosteroid injections on lateral epicondylitis showed that a corticosteroid injection is more effective in the short-term (less 
than 6 weeks) compared to placebo, local anesthetic, and conservative treatments when analyzing pain, global improvement, and grip strength. However, there is no conclusive evidence that suggests that corticosteroid injections are effective in providing long-term improvement of pain [12]. Therefore, a corticosteroid injection is best used for short-term pain relief or in those with debilitating symptoms.

Botulinum toxin injections have also been, used but given that these injections can have the side effect of causing digit paresis and weakness of finger extension [13], this should not be first-line injectable treatment. In a double-blind, placebo-controlled, randomized controlled trial of patients with symptoms lasting longer than three months, patients had a reduction of pain (as reported by a pain VAS, the main outcome measure) in the group receiving botulinum toxin compared to those receiving a saline injection [13]. However, the botulinum toxin group still had pain at 4 and 12 weeks follow-up and were not pain-free. Some authors suggest that if botulinum toxin is going to be used it should be used only on those patients with symptoms for more than 1 year [14].

More recently, research has been done on the efficacy of autologous blood injections around the lateral epicondyle [15]. While there was no control group in this study, some of the patients who had received multiple corticosteroid injections still responded favorably to the treatment. It was postulated that introducing blood to the injured area would provoke the inflammatory cascade and restart the healing process.

Those who do not respond to a focused, supervised, and intense course of several weeks of conservative care, and continue to have debilitating pain, may be considered to have failed nonoperative management. Surgery may be needed for these individuals, though some suggest waiting at least one year before considering this [14].

\section{Operative}

When extensive conservative measures have been pursued, but are unsuccessful, then surgery is considered to alleviate pain and improve function. While there are multiple surgical treatments, the goal is to remove all the abnormal tissues and release any of the residual tension on the remaining extensor wad. Options include open surgery, arthroscopic lateral release, and percutaneous treatment to debride the gray, friable, angiofibrotic tissue [1]. While one review [1] reported that studies have had excellent outcomes in those with lateral epicondylitis, a Cochrane Database Systematic Review did not identify any controlled trials investigating the effect of surgery on tennis elbow pain [16].

\section{General approach to treatment of proximal wrist extensor tendinopathy}

Once someone is diagnosed with proximal wrist extensor tendinopathy, then it is reasonable to modify the patient's activity and pursue physical therapy with eccentric wrist strengthening exercises, wrist extensor stretches, and crossfriction massage. In an athlete or someone with a job position that requires repetitive use of the hands, a proximal forearm strap may be useful to attenuate the symptoms, while allowing patients to continue with their daily activities. The strap is applied circumferentially around the proximal forearm and attempts to offload the injured area of the tendon.

As part of physical therapy any biomechanical or ergonomic issues should be addressed. If the patient is an athlete, then physical therapy should address any deficits that could lead to overloading the elbow, which would include evaluating the kinetic chain and the patient's form during sports play. NSAIDs or other analgesics can be used for pain control. If pain is severe, then an injection with corticosteroid is reasonable. While autologous blood injections or botulinum toxin injections are more controversial, either is reasonable if above measures fail or if the patient does not want surgery. When conservative measures fail, then surgery should be considered.

\section{Conclusion}

Proximal wrist extensor tendinopathy is a condition that can be debilitating, but generally responds well to nonoperative care. While there is a dearth of randomized controlled trials, there is some evidence that manipulation techniques, exercise, acupuncture, and corticosteroids can provide relief. However, it is clear from recent metaanalyses and systematic reviews that more research is needed, with better methodology. Few patients will need to go on to surgery for this condition.

\section{References}

1. Whaley AL, Baker CL. Lateral epicondylitis. Clin Sports Med 2004;23:677-91.

2. Bisset L, Paungmali A, Vincenzino B, Beller E. A systematic review and meta-analysis of clinical trials on physical interventions for lateral epicondylalgia. Br J Sports Med 2005;39:411-22.

3. Shiri R, Viikari-Juntura E, Varonen H, Heliovaara M. Prevalence and determinants of lateral and medial epicondylitis. Am J Epidemiol 2006;164:1065-74.

4. Norris C. Sports injuries: diagnosis and management. Philadelphia: Butterworth Heinemann Publishers; 2004. p. 29-39.

5. Kraushaar BS, Nirschl RP. Current concepts review: tendinosis of the elbow (tennis elbow). J Bone Joint Surg 1999;81-A:259-78. 
6. Magee DJ. Orthopedic physical assessment. Philadelphia: W.B. Saunders Company; 1997. p. 258.

7. Davis TRC. Diagnostic criteria for upper limb disorders in epidemiologic studies. J Hand Surg Br 1998;23B:567-9.

8. Magra M, Maffulli N. Nonsteroidal antiinflammatory drugs in tendinopathy. Clin J Sports Med 2006;16:1-3.

9. Manias P, Stasinopoulos D. A controlled clinical pilot trial to study the effectiveness of ice as a supplement to the exercise programme for the management of lateral elbow tendinopathy. $\mathrm{Br}$ J Sports Med 2006;40:81-5.

10. Woodley BL, Newsham-West RJ, Baxter GD. Chronic tendinopathy: effectiveness of eccentric exercise. Br J Sports Med published online 24 Oct 2006; doi:10.1136/bjsm.2006.29769

11. Fahlstrom M, Jonsson P, Lorentzon R, Alfredson H. Chronic Achilles tendon pain treated with eccentric calf-muscle training. Knee Surg Sports Traumatol Arthrosc 2003;11:327-33.
12. Smidt N, Assendelft WJJ, van der Windt DAWM, Hay EM, Buchbinder R, Bouter LM. Corticosteroid injections for lateral epicondylitis: a systematic review. Pain 2002;96:23-40.

13. Wong SM, Hui ACF, Tong PY, Poon D, Yu E, Wong LKS. Treatment of lateral epicondylitis with botulinum toxin: a randomized, double-blind, placebo-controlled trial. Ann Intern Med 2005;143:793-7.

14. Akuthota V, Chou LH, Drake DF, Nadler SF, Toledo SD. Sports and performing arts medicine. 2. Shoulder and elbow overuse injuries in sports. Arch Phys Med Rehabil 2004;85(3 Suppl 1): S52-8.

15. Edwards SG, Calandruccio JH. Autologous blood injections for refractory lateral epicondylitis. J Hand Surg 2003;28A:272-8.

16. Buchbinder R, Green S, Bell S, Barnsley L, Smidt N, Assendelft WJ. Surgery for lateral elbow pain. Cochrane Database Syst Rev 2002;(1):CD003525. 